\title{
ESTUDO COMPARATIVO DA TOLERÂNCIA À TOXICIDADE DE FERRO E ALUMÍNIO EM ARROZ ( ${ }^{(1)}$
}

\author{
CARLOS EDUARDO DE OLIVEIRA CAMARGO $(2,3)$ \\ e OCTÁVIO BENTO DE ALMEIDA CAMARGO $\left({ }^{2}, 3\right)$
}

\begin{abstract}
RESUMO
Foram estudados onze cultivares de arroz em soluçōes nutritivas, arejadas e não arejadas, contendo diferentes concentrações de ferro e alumínio, mantendo-se constante a temperatura de $30 \pm 1^{\circ} \mathrm{C}$ e o pH das soluções a 4,0 . A tolerância foi medida pelo comprimento médio da raiz primária, peso seco da parte aérea $\mathrm{e}$ raízes de vinte plântulas cultivadas durante dez dias em solução nutritiva contendo diferentes concentrações de ferro e alumínio. Com as concentrações de $0 ; 20 \mathrm{e}$ $40 \mathrm{mg} /$ litro de $\mathrm{Al}^{3+}$ nas soluções com ou sem arejamento, em presença de $0,56 \mathrm{mg} / \mathrm{li}$ tro de $\mathrm{Fe}$, verificou-se, pelo crescimento radicular, que os cultivares IAC-164, IAC-165, IAC-47, IAC-25, IAC-435, IAC-120, Blue Bonnet e Pérola foram tolerantes e, IR-8, IAC-899 e IR-841, sensíveis. Nas soluçōes arejadas sem Al onde se adicionaram $200 \mathrm{mg} /$ ittro de $\mathrm{Fe}$, o 'IAC-164' foi o que apresentou maior crescimento das raízes, diferindo dos cultivares IAC-435, IAC-899, Blue Bonnet, IAC-25, IR-8 e IR-841, mas não dos cultivares IAC-120, IAC-47, IAC-165 e Peróla. Nas soluções não arejadas, o mesmo resultado foi obtido, com exceção do 'IAC-164' que não diferiu do 'IAC-25'. Nas soluçōes arejadas sem Al, contendo $400 \mathrm{mg} /$ litro de $\mathrm{Fe}$, o cultivar mais tolerante foi IAC-164, diferindo somente, porém, dos cultivares IAC-899, IR-841 e IR-8, considerando o crescimento das raízes. Nas soluções não arejadas sem Al empregando-se $400 \mathrm{mg} /$ litro de $\mathrm{Fe}$, não ocorreram diferenças significativas para tolerância entre os cultivares estudados. O 'IAC-164' mostrou grande tolerância à toxicidade de $\mathrm{Fe}$ e $\mathrm{Al}$, mesmo nos tratamentos onde foram empregados $20 \mathrm{mg} /$ litro de $\mathrm{Al}^{3+}$ combinados com $200 \mathrm{mg} /$ litro de $\mathrm{Fe}$, e $40 \mathrm{mg} / \mathrm{litro}$ de $\mathrm{Al}^{3+}$ combinados com $400 \mathrm{mg} /$ litro de $\mathrm{Fe}$. Os cultivares tolerantes ao $\mathrm{Al}$, quando plantados
\end{abstract}

$\left({ }^{1}\right)$ Recebido para publicação em 3 de novembro de 1983.

( ${ }^{2}$ ) Seção de Arroz e Cereais de Inverno, Instituto Agronômico, Caixa Postal 28, 13100 - Campinas (SP).

( $\left.{ }^{3}\right)$ Com bolsa de suplementação do CNPq. 


\begin{abstract}
em soluçôes com $0,56 \mathrm{mg} /$ litro de $\mathrm{Fe}$ e doses crescentes de $\mathrm{Al}$, mostraram que os teores de $\mathrm{Al}$ na matéria seca da parte aérea aumentaram, sobretudo nas soluções arejadas. Nessas condições, os teores de $\mathrm{P}, \mathrm{Ca}, \mathrm{Mg}, \mathrm{Fe}, \mathrm{Cu}, \mathrm{Zn}$ e $\mathrm{Mn}$ tenderam a diminuir e, os de $\mathrm{K}$, a aumentar, à medida que se aumentaram as concentraçōes de $\mathrm{Al}$ nas soluçōes, com ou sem arejamento. Os cultivares sensíveis ao Al mostraram aumento nos teores de $\mathrm{P}, \mathrm{Fe}$ e $\mathrm{Al}$ na matéria seca da parte aérea e redução nos teores dos demais nutrientes, à medida que se elevaram as concentraçōes de $\mathrm{Al}$ nas soluções. Com o aumento nas concentraçōes de Fe nas soluções em ausência de $\mathrm{Al}$, os cultivares estudados mostraram tendência de diminuição dos teores de $\mathrm{Ca}, \mathrm{Mg}, \mathrm{Cu}$ e $\mathrm{Zn}$, na matéria seca da parte aérea e elevação dos de $\mathrm{K} \mathrm{e} \mathrm{Fe}$, com pouca variaçã̃o nos teores de P. Os resultados obtidos demonstraram que os cultivares de arroz estudados poderiam ser diferenciados em relação à tolerância à toxicidade de $\mathrm{Fe}$ e $\mathrm{Al}$, desde que concentraçōes adequadas desses elementos fossem utilizadas nas soluçôes nutritivas, com ou sem arejamento.
\end{abstract}

Termos de indexação: toxicidade de ferro e de alumínio em arroz; soluções arejadas e não-arejadas; tolerância; sensibilidade.

\title{
1. INTRODUÇÃO
}

Estudos conduzidos em solo possibilitaram a diferenciação dos cultivares de arroz tolerantes, moderadamente tolerantes e sensíveis à toxicidade por ferro (ANNUAL ..., 1972, 1973, 1974, 1980). Entre os tolerantes, destacaram-se IR-1552, IR-20, IR-36, IR-42, IR-43, IR-44, Mat Candu e Pokkali e, entre os sensíveis, IR-5, IR-8, IR-759-79-2, ICA-10, Monolaya e Purbachi.

CAMARGO (1984), estudando sete cultivares de arroz em soluções nutritivas não arejadas contendo quatro níveis de ferro $(0,56,100$, 200 e $400 \mathrm{mg} /$ litro), mantendo constante a temperatura de $30 \pm 1^{\circ} \mathrm{C}$ e o pH das soluçôes igual a 4,0, concluiu que o 'Pérola' mostrou tolerância à presença de doses crescentes de ferro nas soluções, os cultivares IAC-435 e IR-8 foram sensíveis, e IAC-120, IAC-899, IR-841 e Blue Bonnet apresentaram reações intermediárias. Concluiu também que a maior tolerância do 'Pérola' pareceu ser devida a menor absorção de ferro das soluções.

Milhões de hectares nos trópicos são representados por solos ácidos, cuja toxicidade, causada pela presença do alumínio, é uma das principais causas da baixa produtividade das culturas. Logo, a obtenção de cultivares portadores de tolerância ao alumínio seria de alto interesse nos diferentes programas de melhoramento dessas regiões (MARTINES, 1979).

Os cultivares de arroz IAC-899, IR-8 e IR-841 foram sensíveis a $10 \mathrm{mg} /$ litro de alumínio quando cultivados durante dez dias em soluções nutritivas arejadas, com $\mathrm{pH}=4,0$ e temperatura de $30 \pm 1^{\circ} \mathrm{C}$, e IAC-435, IAC-120, IAC-47, IAC-25, IAC-164, IAC-165, Pérola e Blue Bonnet foram tolerantes nas mesmas condições (CAMARGO, 1984). 
Considerando as possíveis interações existentes nos solos ácidos entre ferro e alumínio, o presente trabalho tem por objetivo estudar o comportamento de cultivares de arroz em soluções nutritivas, com e sem arejamento, contendo diferentes concentrações de ferro e de alumínio, visando à detectação de fontes de tolerância para uso futuro em programa de melhoramento genético.

\section{MATERIAL E METTODOS}

No delineamento estatístico empregado, parcelas subdivididas com duas repetições, as parcelas foram compostas por sete soluções de tratamento com diferentes concentrações de ferro e alumínio, e as subparcelas, por onze cultivares de arroz. Instalaram-se dois experimentos, um com as soluções arejadas e o outro sem arejamento, estudando-se os cultivares seguintes: IAC-435, IAC-120, IAC-47, IAC-165, Pérola, IAC-899, Blue Bonnet, IAC-164, IAC-25, IR-8 e IR-841.

As sementes dos onze cultivares de arroz, cuidadosamente lavadas com uma solução de hipoclorito de sódio a $10 \%$ foram colocadas para germinar em caixas de Petri por 48 horas. Após esse tempo, as radículas estavam iniciando a emergência.

Foram escolhidas 20 sementes uniformes de cada cultivar e colocadas sobre o topo de 28 telas de náilon adaptadas a 28 vasilhas plásticas de 8,30 litros de capacidade contendo soluções nutritivas, de maneira que as sementes fossem mantidas úmidas e as radículas emergentes tocassem nas soluções, tendo, portanto, um pronto suprimento de nutrientes.

A composição da solução nutritiva completa foi a seguinte: $\mathrm{Ca}\left(\mathrm{NO}_{3}\right)_{2} 4 \mathrm{mM} ; \mathrm{MgSO}_{4} 2 \mathrm{mM} ; \mathrm{KNO}_{3} 4 \mathrm{mM} ;\left(\mathrm{NH}_{4}\right)_{2} \mathrm{SO}_{4} 0,435 \mathrm{mM}$; $\mathrm{KH}_{2} \mathrm{PO}_{4} 0,5 \mathrm{mM} ; \mathrm{MnSO}_{4} 2,0 \mu \mathrm{M} ; \mathrm{CuSO}_{4} 0,3 \mu \mathrm{M} ; \mathrm{ZnSO}_{4} 0,8 \mu \mathrm{M} ; \mathrm{NaCl}$ $30 \mu \mathrm{M}$; Fe-CYDTA $10 \mu \mathrm{M} ; \mathrm{Na}_{2} \mathrm{MoO}_{4} 0,1 \mu \mathrm{M}$ e $\mathrm{H}_{3} \mathrm{BO}_{3} 10 \mu \mathrm{M}$. O pH das soluções foi ajustado para 4,0 com solução de $\mathrm{H}_{2} \mathrm{SO}_{4} 1 \mathrm{~N}$. As vasilhas plásticas contendo as soluções foram colocadas em banho-maria com temperatura de $30 \pm 1^{\circ} \mathrm{C}$ dentro do laboratório, sendo o experimento mantido com luz artificial em sua totalidade.

Decorridas 48 horas nessas condições, cada uma das 28 telas de náilon contendo vinte plântulas dos onze cultivares foi dividida em dois grupos de quatorze, constituindo cada grupo um experimento, e transferida para vasilhas plásticas contendo as soluções de tratamento. No primeiro experimento, as soluções de tratamento não foram arejadas e, no outro, o arejamento das soluções foi mantido durante todo o período. 
A composição das soluções de tratamento foi um décimo da solução nutritiva completa, porém com as seguintes diferenças:

1. $0 \mathrm{mg} /$ litro de $\mathrm{Al}^{3+}$ e $0,56 \mathrm{mg} /$ litro de $\mathrm{Fe}^{2+}$;

2. $20 \mathrm{mg} /$ litro de $\mathrm{Al}^{3+}$ e $0,56 \mathrm{mg} /$ litro de $\mathrm{Fe}^{2+}$;

3. $40 \mathrm{mg} /$ litro de $\mathrm{Al}^{3+}$ e $0,56 \mathrm{mg} /$ litro de $\mathrm{Fe}^{2+}$;

4. $0 \mathrm{mg} /$ litro de $\mathrm{Al}^{3+}$ e $200 \mathrm{mg} /$ litro de $\mathrm{Fe}^{2+}$;

5. $0 \mathrm{mg} /$ litro de $\mathrm{Al}^{3+}$ e $400 \mathrm{mg} /$ litro de $\mathrm{Fe}^{2+}$;

6. $20 \mathrm{mg} /$ litro de $\mathrm{Al}^{3+}$ e $200 \mathrm{mg} /$ litro de $\mathrm{Fe}^{2+}$;

7. $40 \mathrm{mg} /$ litro de $\mathrm{Al}^{3+}$ e $400 \mathrm{mg} /$ litro de $\mathrm{Fe}^{2+}$.

Como fonte de alumínio, foi utilizado $\mathrm{Al}_{2}\left(\mathrm{SO}_{4}\right)_{3} .18 \mathrm{H}_{2} \mathrm{O}$ e, como fonte de ferro, $\mathrm{FeSO}_{4} .7 \mathrm{H}_{2} \mathrm{O}$.

As plantas, após crescerem nas soluções de tratamento durante dez dias, com o $\mathrm{pH}$ mantido o mais próximo de 4,0 , por ajustamentos diários, foram retiradas, determinando-se o crescimento da raiz primária de cada plântula, em milímetro. A seguir, foram separadas as raízes das partes aéreas das vinte plântulas de cada cultivar submetido a crescimento em soluções contendo diferentes concentrações de ferro e alumínio. As partes aéreas e as raízes de cada cultivar foram colocadas para secar em estufa com temperatura de $45^{\circ} \mathrm{C}$, por cinco dias, determinando-se os respectivos pesos secos. As partes aéreas secas de cada tratamento foram analisadas quimicamente quanto aos teores de $\mathrm{P}, \mathrm{K}, \mathrm{Ca}, \mathrm{Mg}, \mathrm{Fe}, \mathrm{Cu}, \mathrm{Mn}, \mathrm{Zn}$ e $\mathrm{Al}$, segundo o método de BATAGLIA et alii (1978).

Os dados foram analisados estatisticamente, considerando a média do crescimento das raízes das vinte plântulas de cada cultivar após a influência prejudicial de diferentes concentrações de ferro e alumínio. As comparações entre as médias de comprimento de raiz das vinte plântulas de cada cultivar dentro de uma mesma concentração de ferro e alumínio foram feitas pelo teste de Tukey.

\section{RESULTADOS E DISCUSSÃO}

O comprimento médio das raízes de vinte plântulas de onze cultivares de arroz medidos após dez dias de crescimento em soluções nutritivas arejadas e não arejadas, contendo diferentes concentrações de ferro e alumínio encontram-se no quadro 1.

A figura 1 apresenta os sistemas radiculares dos cultivares IAC-164, IAC-435 e IAC-899 após dez dias de crescimento em soluções arejadas contendo diferentes concentrações de $\mathrm{Fe}$ e $\mathrm{Al}$ e, a figura 2, os crescimentos das 


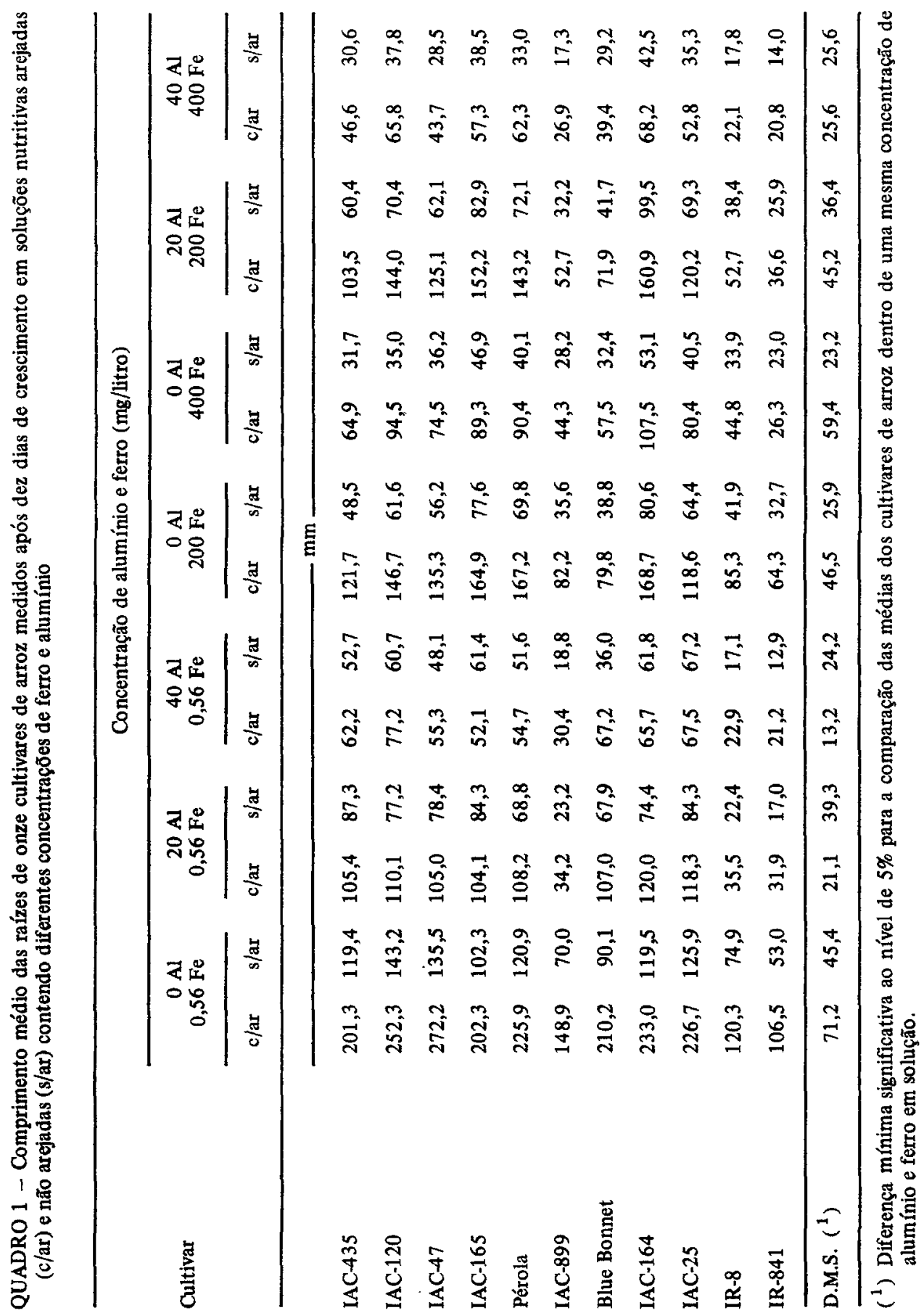




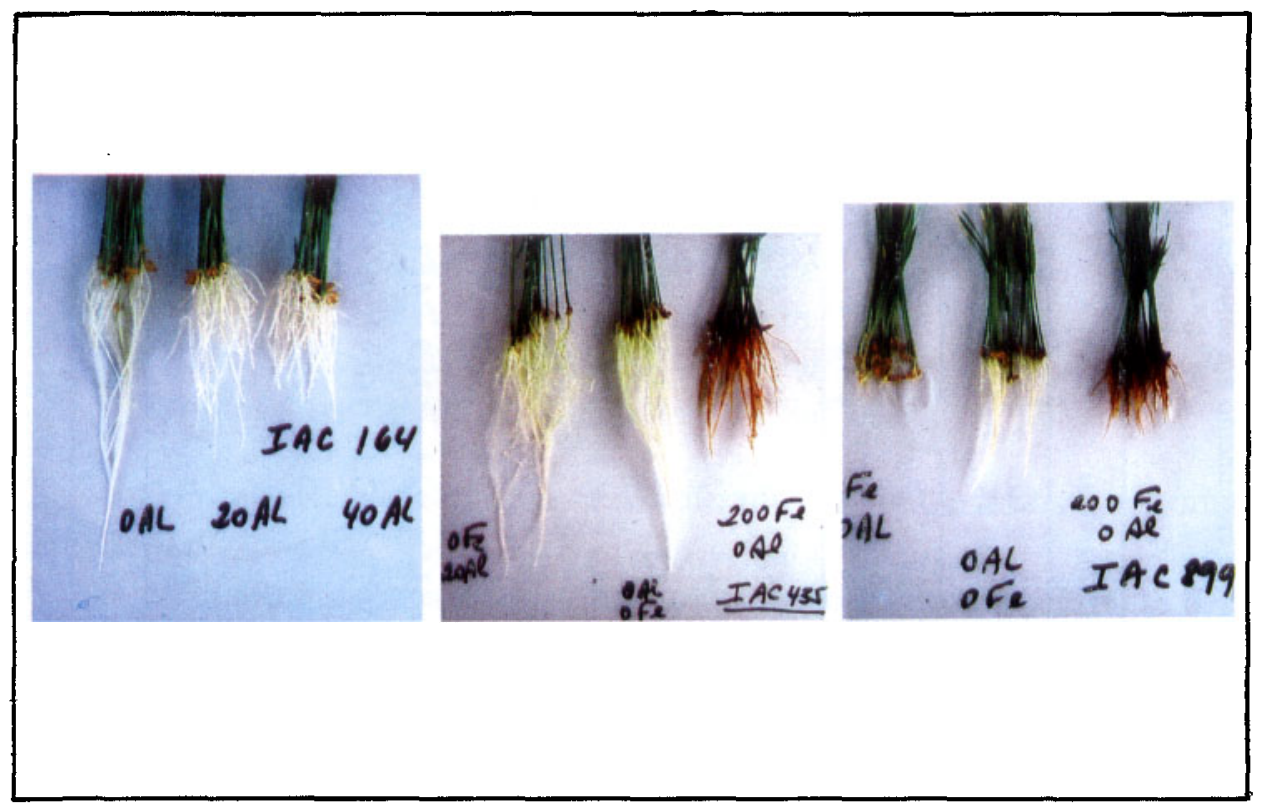

FIGURA 1 - A: Sistema radicular do cultivar de arroz IAC-164, após dez dias de desenvolvimento em solução nutritiva arejada contendo 0,20 e $40 \mathrm{mg} /$ litro de $\mathrm{Al}$; B: Sistema radicular do cultivar de arroz IAC435 após dez dias de desenvolvimento em solução nutritiva arejada contendo $20 \mathrm{mg} / \mathrm{litro}$ de $\mathrm{Al}$ e $0,56 \mathrm{mg} / \mathrm{litro}$ de $\mathrm{Fe}, 0 \mathrm{mg} / \mathrm{litro}$ de Al e $0,56 \mathrm{mg} / \mathrm{litro}$ de $\mathrm{Fe}, 0 \mathrm{mg} / \mathrm{litro}$ de $\mathrm{Al}$ e $200 \mathrm{mg} / \mathrm{litro}$ de $\mathrm{Fe}$; C: Sistema radicular do cultivar de arroz IAC-899 após dez dias de desenvolvimento em solução nutritiva arejada contendo $20 \mathrm{mg} /$ litro de $\mathrm{Al}$ e $0,56 \mathrm{mg} / \mathrm{lit}$ to de $\mathrm{Fe}, 0 \mathrm{mg} / \mathrm{litro}$ de $\mathrm{Al}$ e $0,56 \mathrm{mg} / \mathrm{litro}$ de Fe, Omg/litro de Al e 200mg/litro de Fe.

raízes dos cultivares IAC-165, IAC-899, IAC-435 e Pérola, estudados em soluções nutritivas com e sem arejamento contendo diferentes concentrações de Fe e Al.

O crescimento radicular de todos os cultivares estudados foi senstvelmente maior em soluções arejadas do que nas sem arejamento, independentemente das concentrações de $\mathrm{Fe}$ e $\mathrm{Al}$ presentes nas soluçōes.

Considerando o crescimento das raízes em solução arejada, contendo $0 \mathrm{mg} /$ litro de $\mathrm{Al}$ e $0,56 \mathrm{mg} /$ litro de $\mathrm{Fe}$, verificou-se que o 'IAC-47' foi o que exibiu maior valor, diferindo significativamente dos cultivares IAC-899, IR-8 e IR-841, porém não dos demais. O mesmo resultado foi obtido quando nas soluções não arejadas. Como os cultivares IR-8, IAC-899 e IR-841 são originários das Filipinas e selecionados para condições de irrigação, mos- 

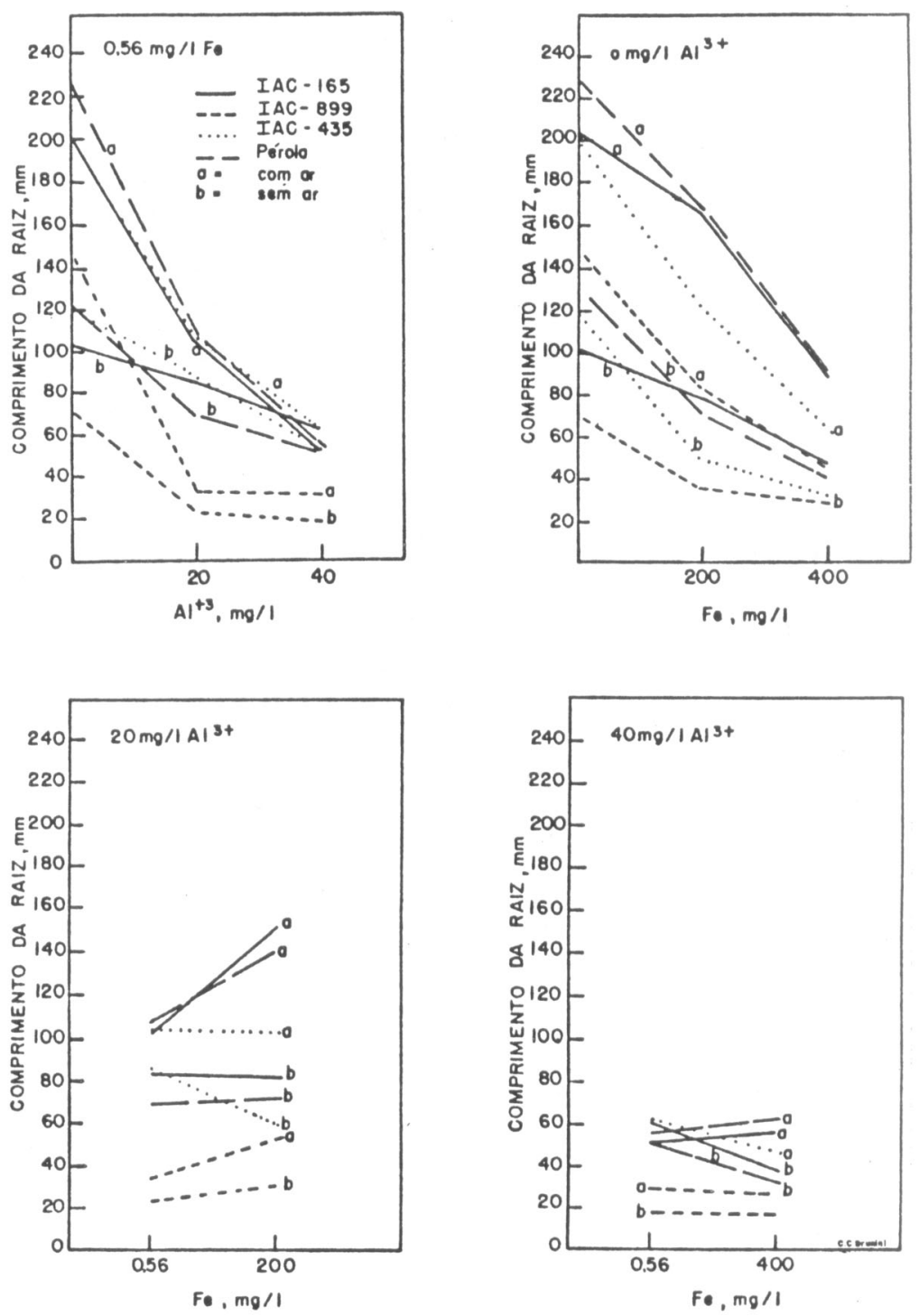

FIGURA 2 - Efeitos das diferentes concentraçōes de alumínio e ferro no crescimento da raiz de quatro cultivares de arroz estudados em soluções nutritivas arejadas (com ar) e não arejadas (sem ar). 
traram um crescimento radicular significativamente menor do que os nacionais, principalmente em relação ao 'IAC-47', selecionado para condições de cultivo em sequeiro.

Quando se adicionaram $20 \mathrm{mg} /$ litro de $\mathrm{Al}$ e $0,56 \mathrm{mg} /$ litro de Fe na solução tratamento arejada, o cultivar indicado para condições de plantio em sequeiro mostrou raizes mais compridas, diferindo dos cultivares IR-841, IAC-899 e IR-8 que foram bastante sensíveis ao alumínio, porém não dos demais. Nas soluções não arejadas, o 'IAC-435', selecionado para condições de várzeas com altos teores de alumínio, foi o mais tolerante, diferindo estatisticamente de IR-8, IR-841 e IAC-899.

O 'IAC-120' foi o que mostrou raízes mais compridas nas soluções arejadas contendo $40 \mathrm{mg} /$ litro de $\mathrm{Al}$ e $0,56 \mathrm{mg} / \mathrm{litro}$ de $\mathrm{Fe}$, diferindo significativamente dos cultivares IAC-435, IAC-47, IAC-165, Pérola, IAC899, IR-8 e IR-841, porém não do 'Blue Bonnet', 'IAC-25' e 'IAC-164'. Em solução não arejada, o 'IAC-25' foi o que mostrou as raízes com maior crescimento, diferindo dos cultivares Blue Bonnet, IAC-899, IR-841 e IR-8.

Os resultados mostraram que quando foi mantido constante o nível de $0,56 \mathrm{mg} /$ litro de Fe nas soluções arejadas e não arejadas e foi aumentada a concentração de $\mathrm{Al}$ de 0 para $40 \mathrm{mg} /$ litro, os cultivares IAC-47, IAC-120, IAC-435, IAC-165, Pérola, IAC-164 e IAC-25 foram os mais tolerantes, e, IR-8, IAC-899 e IR-841, sensíveis.

Considerando o crescimento radicular nas soluções arejadas contendo $0 \mathrm{mg} /$ litro de $\mathrm{Al}$ e $200 \mathrm{mg}$ /litro de $\mathrm{Fe}$, observou-se que o 'IAC-164' foi o que apresentou maior valor, diferindo pelo teste de Tukey a $5 \%$ dos cultivares IAC-435, IAC-899, Blue Bonnet, IAC-25, IR-8 e IR-841, porém não diferindo do IAC-120, IAC-47, IAC-165 e Pérola. Nas soluções não arejadas, O 'IAC-164' exibiu as raízes mais compridas, diferindo significativamente do IAC-435, IAC-899, Blue Bonnet, IR-8 e IR-841.

Nas soluções de tratamento arejadas contendo $0 \mathrm{mg} /$ litro de $\mathrm{Al}$ e $400 \mathrm{mg} /$ litro de $\mathrm{Fe}$, verificou-se que o cultivar que apresentou maior crescimento do sistema radicular foi 'IAC-164', diferindo estatisticamente, porém, apenas dos cultivares IAC-899, IR-841 e IR-8. Nas soluções sem arejamento, o nível de $400 \mathrm{mg} / \mathrm{litro}$ de Fe provocou toxicidade em todos os cultivares, que não diferiram significativamente entre si.

Nas soluções arejadas contendo $20 \mathrm{mg} /$ litro de $\mathrm{Al}$ e $200 \mathrm{mg} /$ litro de $\mathrm{Fe}$, o 'IAC-164' exibiu as raízes mais compridas, diferindo dos cultivares IAC-435, IAC-899, Blue Bonnet, IR-8 e IR-841, não diferindo, porém, de 'IAC-120', 'IAC-47', 'IAC-165', 'Pérola' e 'IAC-25'. Quando se empregaram soluções não arejadas, 'IAC-164', o mais adaptado a essas condições, diferiu somente dos cultivares IAC-435, IAC-47, IAC-899, Blue Bonnet, IR-8 e IR-841. 
Considerando-se soluções arejadas que receberam $20 \mathrm{mg} /$ litro de Al combinadas com 0,56 e $200 \mathrm{mg} /$ litro de Fe verificou-se uma tendência de aumento no comprimento das raízes quando se aumentou a concentração de ferro: logo, os resultados indicaram que a presença de doses maiores de $\mathrm{Fe}$ na solução diminuíram o efeito da toxicidade de Al. Nas soluções não arejadas não se observou essa tendência, sugerindo que o ferro, nessas condiçōes, seria mais prejudicial às plantas.

Nas soluções arejadas contendo $40 \mathrm{mg} /$ litro de Al e $400 \mathrm{mg} /$ litro de $\mathrm{Fe}$, não se verificaram diferenças entre os cultivares estudados, que se mostraram sensíveis a tais concentrações, porém nas não arejadas, o 'IAC-164' apresentou raízes mais compridas que os demais, diferindo significativamente, porém, só do 'IR-841'.

Nos quadros 2 e 3 encontram-se, respectivamente, os pesos secos das partes aéreas e das raízes das vinte plantas dos onze cultivares estudados em soluções nutritivas arejadas ou não arejadas contendo diferentes concentrações de Fe e Al.

Nos cultivares estudados em soluções nutritivas arejadas ou não, contendo $0,56 \mathrm{mg} /$ litro de $\mathrm{Fe}$ e com concentraçðes de $\mathrm{Al}$ variando de 0,20 e $40 \mathrm{mg} /$ litro, o peso seco da parte aérea decresceu rapidamente para os cultivares sensíveis ao alumínio, tais como IAC-899, IR-8 e IR-841, ao passo que, nos demais, em alguns casos, o peso seco aumentou na dosagem de $20 \mathrm{mg} /$ litro de $\mathrm{Al}$ e depois diminuiu ou decresceu lentamente, à medida que se elevaram as concentrações de alumínio.

Todos os cultivares de arroz tiveram diminuído o peso seco da parte aérea quando se considerou a concentração de $0 \mathrm{mg} /$ litro de alumínio nas soluções arejadas combinadas com níveis crescentes de ferro. Naquelas não arejadas, todos os cultivares diminuíram os pesos secos da parte aérea à medida que se aumentaram as concentrações de ferro, mantendo-se fixa a dosagem de $0 \mathrm{mg} /$ litro de $\mathrm{Al}$, com exceção do 'Pérola', cujo peso seco aumentou com a concentração de $200 \mathrm{mg} /$ litro de $\mathrm{Fe}$ e diminuiu muito pouco com a de $400 \mathrm{mg} /$ litro de $\mathrm{Fe}$, confirmando trabalho anteriormente realizado que mostrou ser este cultivar tolerante à toxicidade de $\mathrm{Fe}$ (CAMARGO, 1984).

Considerando as soluções com ou sem arejamento contendo 200 ou $400 \mathrm{mg} /$ litro de $\mathrm{Fe}$, quando se adicionaram 20 ou $40 \mathrm{mg} /$ litro de $\mathrm{Al}$, houve redução no peso da matéria seca da parte aérea de todos os cultivares, com exceção de Blue Bonnet, mostrando a existência de uma interação positiva entre a toxicidade de ferro e a de alumínio.

$\mathrm{O}$ parâmetro peso seco das raízes tendeu a aumentar à medida que foram adicionadas quantidades de $\mathrm{Al}$ e $\mathrm{Fe}$ nas soluções com ou sem areja- 


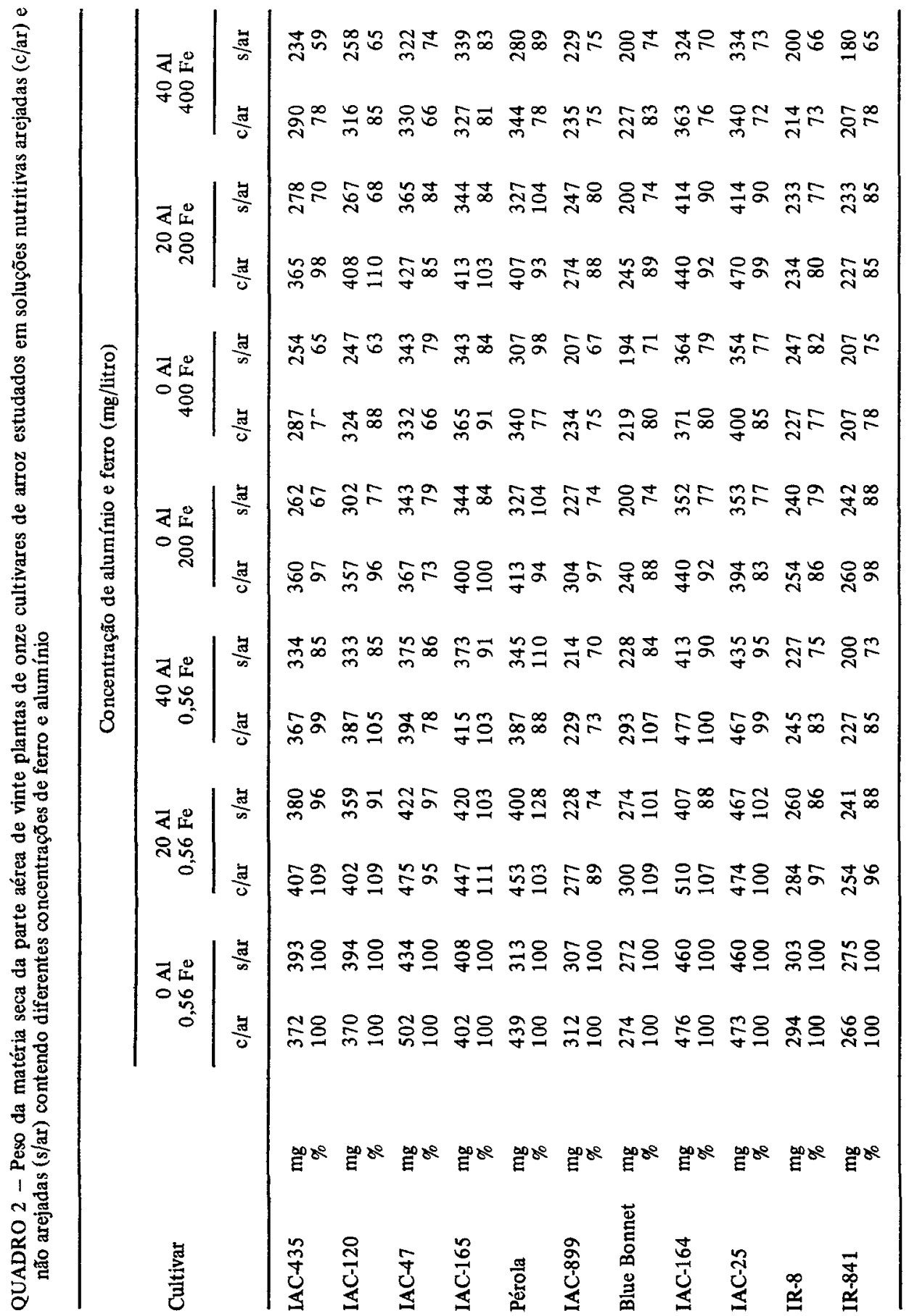




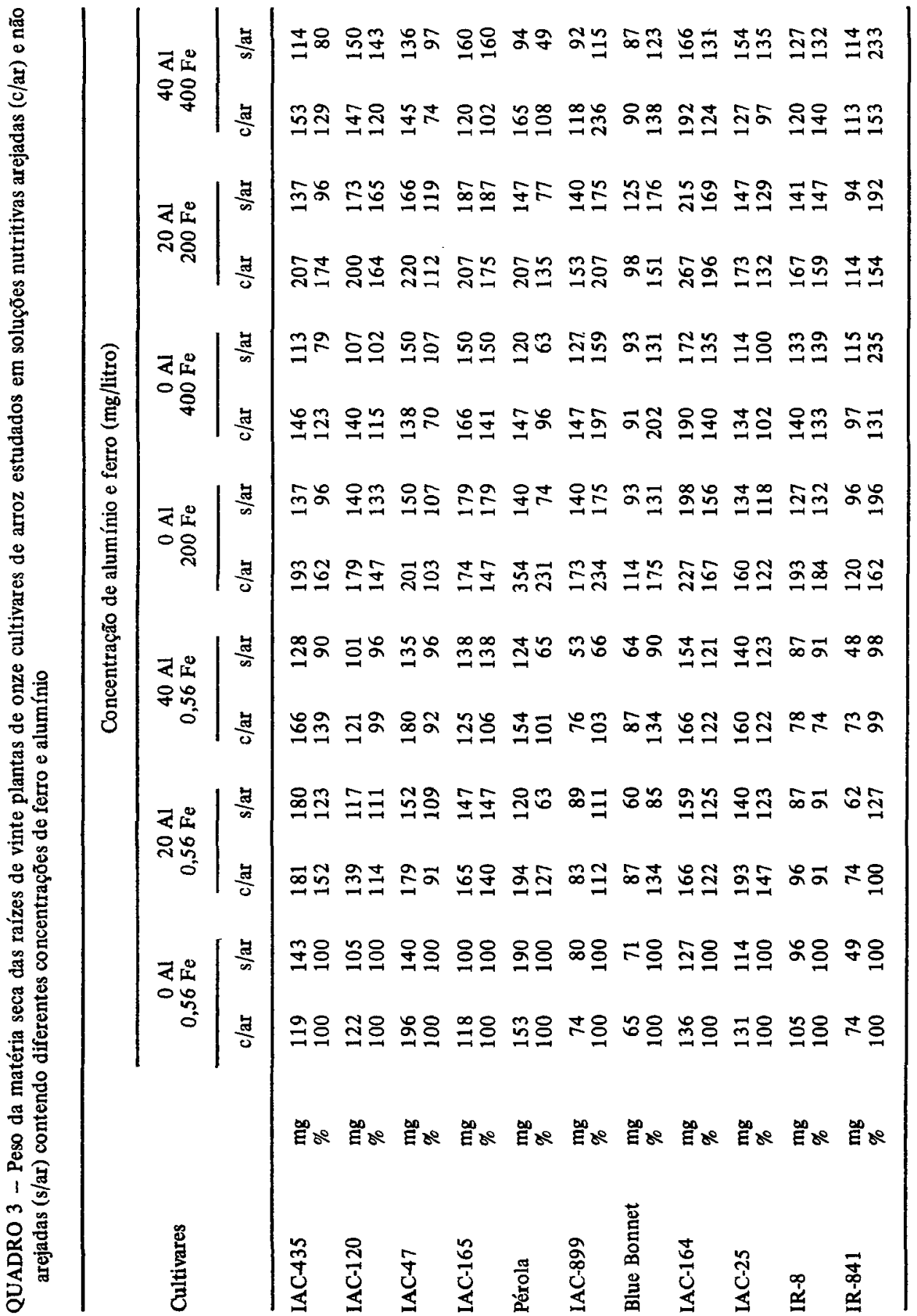


mento para todos os cultivares. Como o excesso de $\mathrm{Fe}$ e $\mathrm{Al}$ torna as raízes mais curtas e grossas e, em muitos casos, mais pesadas que as normais, longas e finas, esse parâmetro não seria bom indicador de tolerância de cultivares de arroz a altas concentraçoes de ferro e alumínio.

A figura 3 apresenta os efeitos das diferentes concentrações de $\mathrm{Fe}$ e Al nas soluções com ou sem arejamento no peso seco total (parte aérea mais raízes) de vinte plantas dos cultivares IAC-165, IAC-899, IAC-435 e Pérola. Verifica-se que 'IAC-165', 'IAC-435' e 'Pérola', tolerantes à toxicidade de $\mathrm{Al}^{3+}$, aumentaram o peso seco total com a concentração de $20 \mathrm{mg} /$ litro de Al nas soluções arejadas ou não, mantendo-se fixa a concentração de $0,56 \dot{\mathrm{mg}} / \mathrm{litro}$ de $\mathrm{Fe}$. Com a concentração de $40 \mathrm{mg} / \mathrm{litro}$ de $\mathrm{Al}$, houve uma redução na matéria seca total. O 'IAC-899', sensível à toxicidade de $\mathrm{Al}$, diminuiu o peso seco total à medida que se adicionaram $20 \mathrm{mg} /$ litro de $\mathrm{Al}$ e continuou a diminuir com a adição de $40 \mathrm{mg} /$ litro de $\mathrm{Al}$.

Nas soluções arejadas com $0 \mathrm{mg} /$ litro de $\mathrm{Al}$, adicionando-se 0,56 ; 200 e $400 \mathrm{mg} /$ litro de Fe, os cultivares Pérola, IAC-165, IAC-435 e IAC-899 aumentaram o peso seco total com o tratamento de $200 \mathrm{mg} / \mathrm{litro}$ de $\mathrm{Fe}$ em relação ao que recebeu $0,56 \mathrm{mg} /$ litro de $\mathrm{Fe}$ e diminuíram com o de $400 \mathrm{mg} /$ litro de Fe. Considerando a mesma situação, porém com soluções não arejadas, o 'IAC-435' foi bastante sensível à concentração de $200 \mathrm{mg} /$ litro de $\mathrm{Fe}$, reduzindo acentuadamente o peso seco total quando comparado com 'IAC165', 'Pérola' e 'IAC-899'.

Em soluções com $20 \mathrm{mg} /$ litro de $\mathrm{Al}$, quando foram adicionados 0,56 e $200 \mathrm{mg} /$ litro de $\mathrm{Fe}$ com ou sem arejamento, verificou-se uma redução no peso seco total do 'IAC-165', 'Pérola' e 'IAC-435', mu' to mais acentuada no 'IAC-435'. Por outro lado, o 'IAC-899', sensível ao Al quando se adicionaram $200 \mathrm{mg} /$ litro de $\mathrm{Fe}$, mostrou um aument. uignificati vo na produção de matéria seca total, sugerindo interação negativa entre $\mathrm{Fe} e \mathrm{Al}$. 's mesmas observaçōes foram obtidas quando se compararam os cultivares IC-435, IAC165, Pérola e IAC-899 em soluçðes de tratamento contendo $0 \mathrm{mg} /$ litro de Al na presença ou na ausência de $400 \mathrm{mg} /$ litro de Fe.

Os teores de $\mathrm{P}, \mathrm{K}, \mathrm{Ca}, \mathrm{Mg}, \mathrm{Fe}, \mathrm{Mn}, \mathrm{Zn}, \mathrm{Cu}$ e $\mathrm{Al}$ na matéria seca da parte aérea dos cultivares IAC-435, IAC-165 e IAC-899, cultivados em soluções nutritivas arejadas e não arejadas contendo diferentes concentrações de $\mathrm{Fe}$ e $\mathrm{Al}$, encontram-se no quadro 4 e, os referentes aos cultivares Pérola, IR-8 e IAC-120, no quadro 5.

As concentrações de $\mathrm{Al}$ e $\mathrm{Fe}$ na matéria seca da parte aérea dos cultivares IAC-165, IAC-899, IAC-435 e Pérola estudados em soluções nutritivas arejadas e não arejadas estão representadas na figura 4.

Os cultivares tolerantes ao alumínio IAC-435, Pérola, IAC-165 e IAC-120, quando estudados em soluções nutritivas contendo $0,56 \mathrm{mg} /$ litro 

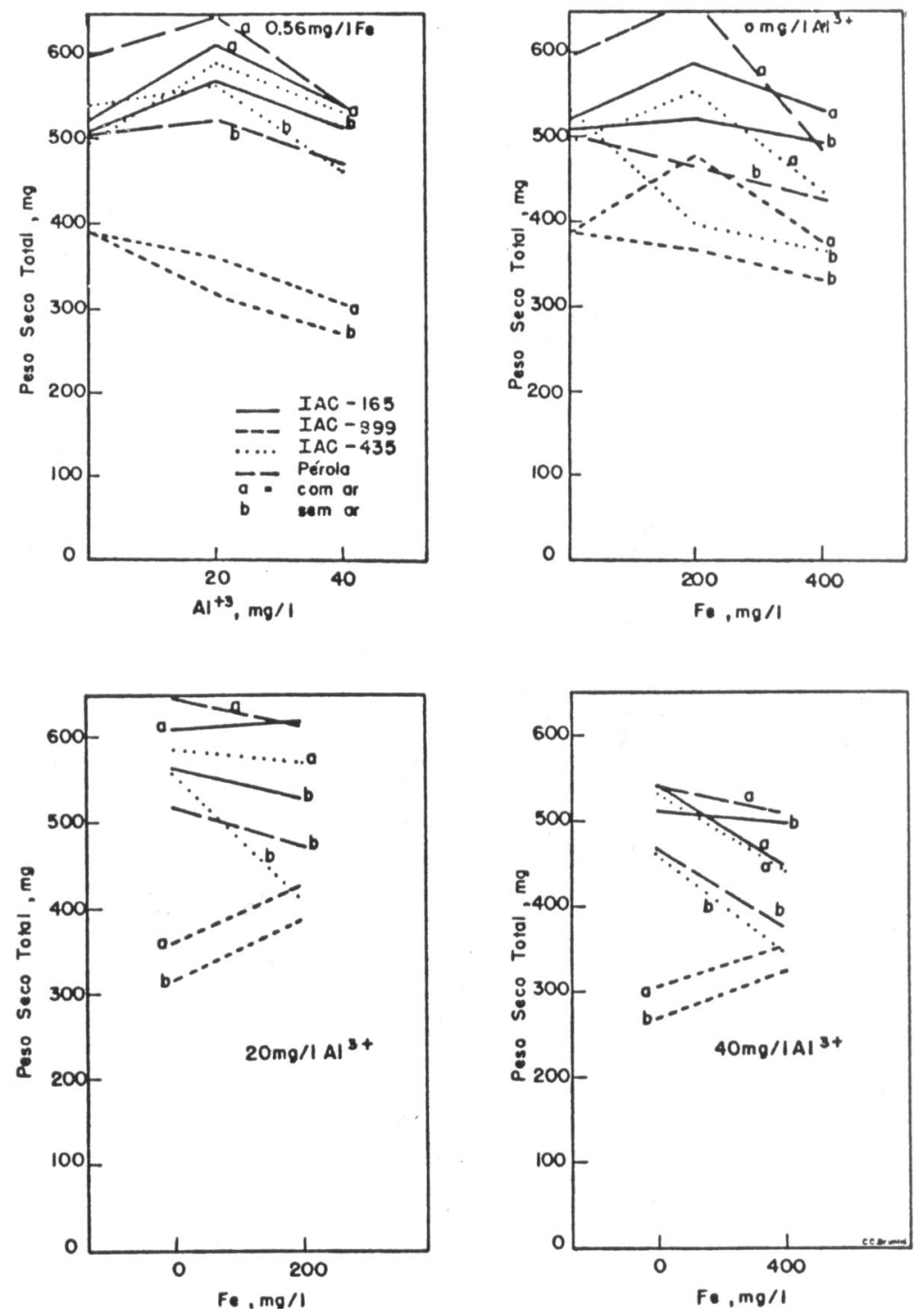

FIGURA 3 - Efeitos das diferentes concentraçōes de ferro e alumínio no peso total (parte área mais raízes) de vinte plantas de quatro cultivares de arroz estudados em soluções nutritivas arejadas (com ar) e não-arejadas (sem ar). 


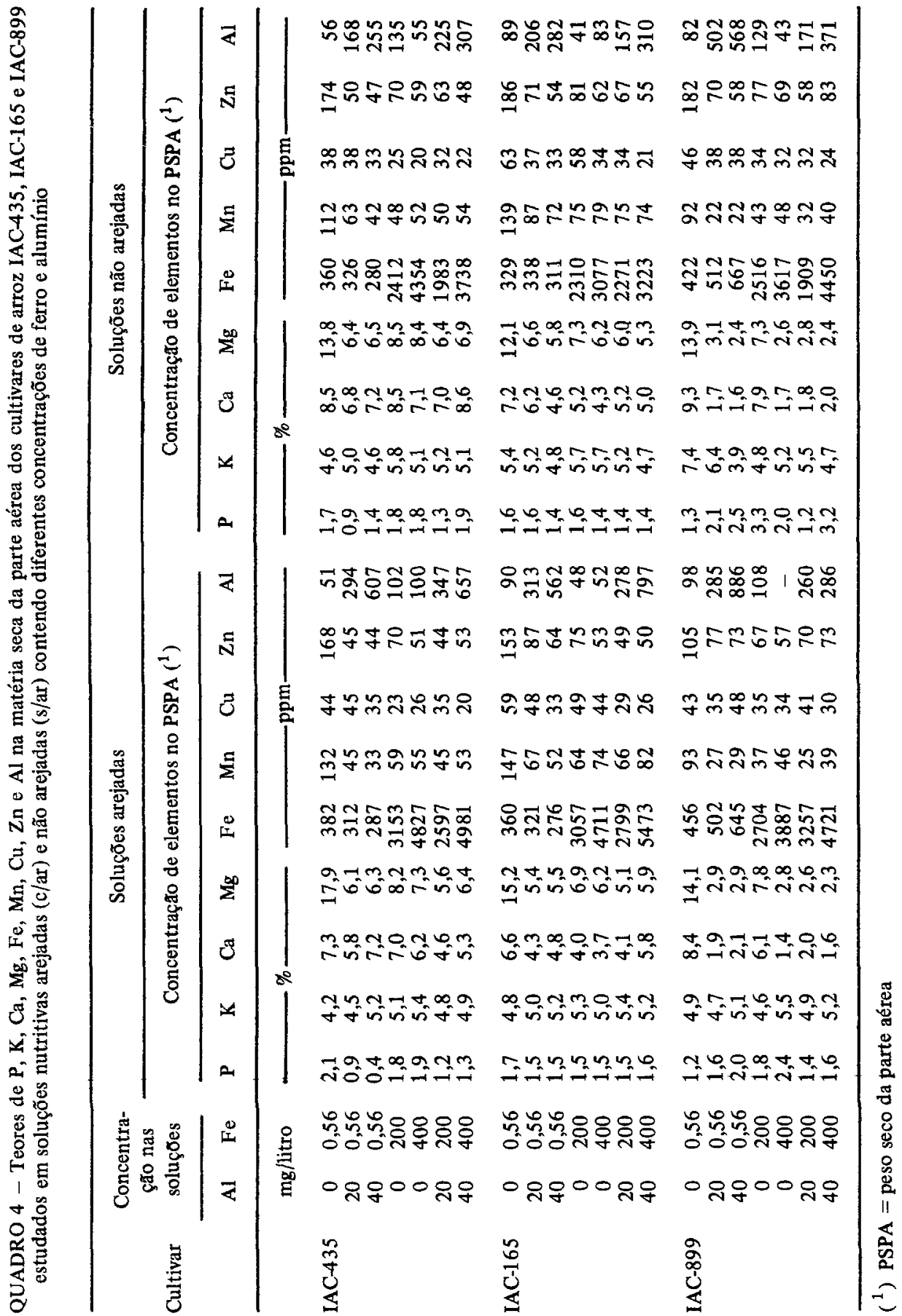




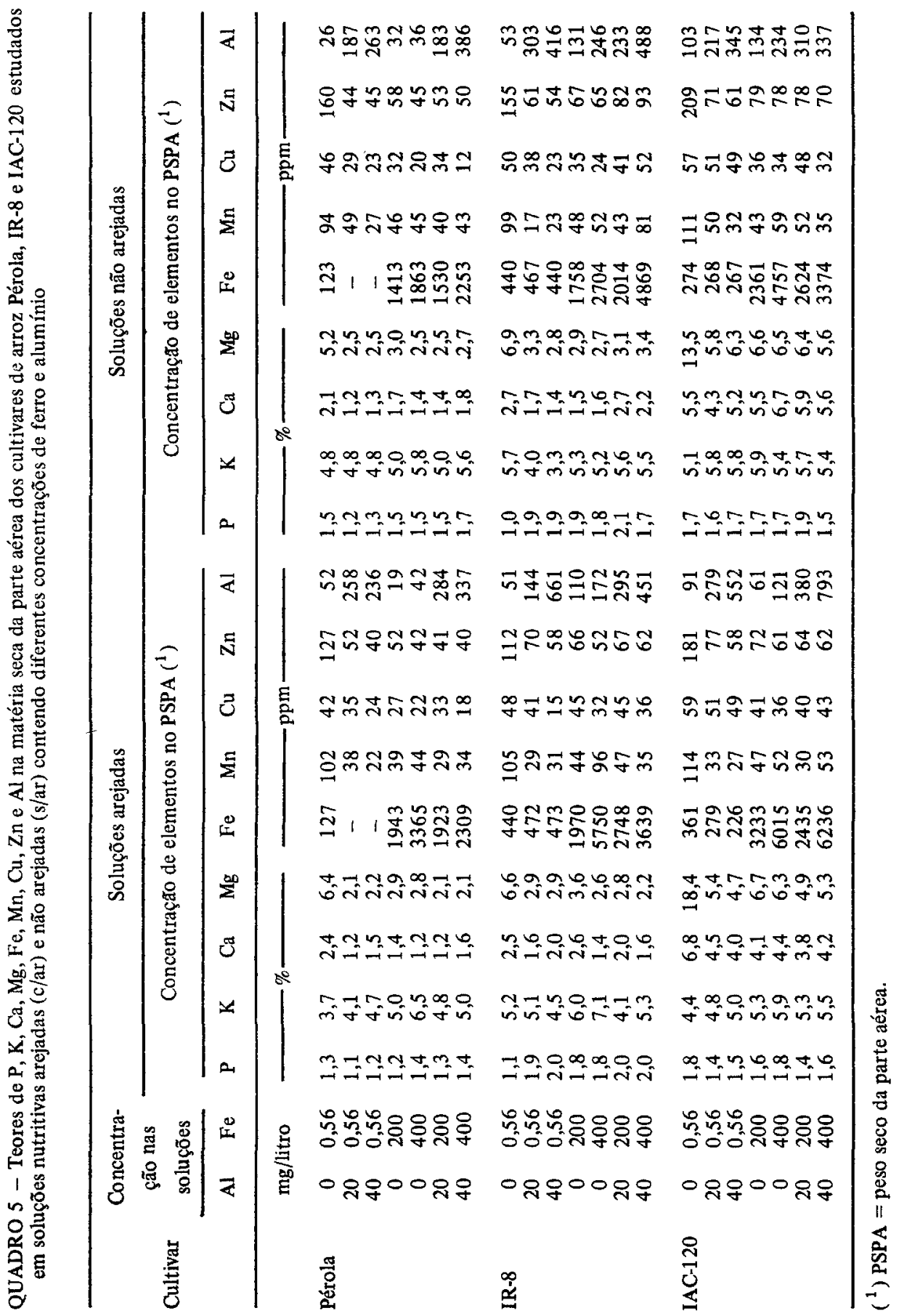



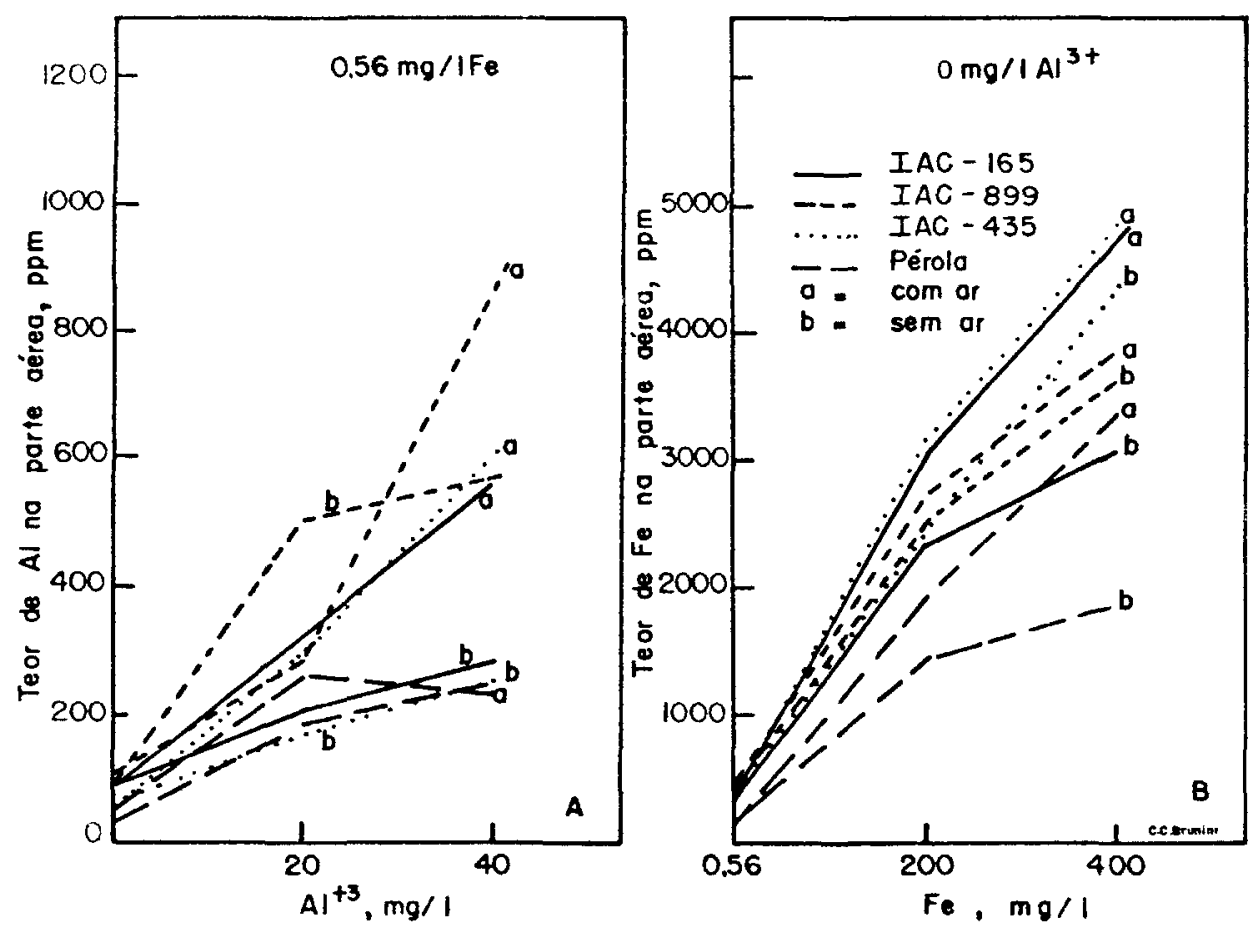

FIGURA 4 - A: Concentrações de $\mathrm{Al}^{3+}$ na matéria seca da parte aérea de quatro cultivares de arroz estudados em soluções nutritivas arejadas (com ar) e não arejadas (sem ar) contendo três diferentes concentrações de alumínio. B: Concentração de ferro na matéria seca da parte aérea de quatro cultivares de arroz estudados em soluções nutritivas arejadas (com ar) e não arejadas (sem ar) contendo três diferentes concentraçôes de ferro.

de $\mathrm{Fe}$ e doses crescentes de $\mathrm{Al}$, mostraram que os teores de $\mathrm{Al}$ na matéria seca da parte aérea aumentaram, sobretudo nas soluções arejadas. Os teores de $\mathrm{P}, \mathrm{Ca}, \mathrm{Mg}, \mathrm{Fe}, \mathrm{Cu}, \mathrm{Zn}$ e $\mathrm{Mn}$ tenderam a diminuir e, os de $\mathrm{K}$, a aumentar à proporção que se elevaram as concentrações de $\mathrm{Al}$ nas soluções com ou sem arejamento. Os cultivares sensíveis ao Al, IR-8 e IAC-899, mostraram aumento nos teores de $\mathrm{P}, \mathrm{Fe}$ e $\mathrm{Al}$ na matéria seca da parte aérea e diminuição nos de $\mathrm{K}, \mathrm{Mg}, \mathrm{Ca}, \mathrm{Mn}, \mathrm{Cu}$ e $\mathrm{Zn}$, conforme se aumentou a concentração de Al nas soluções.

Nas soluções sem Al, porém com doses crescentes de Fe, todos os cultivares considerados mostraram tendência de diminuição dos teores de $\mathrm{Ca}, \mathrm{Mg}, \mathrm{Mn}, \mathrm{Cu}$ e $\mathrm{Zn}$ na matéria seca da parte aérea, e aumento dos de $\mathrm{Ke}$ $\mathrm{Fe}$, sendo que pouco efeito se observou nos teores de P. O cultivar Pérola, 
considerado tolerante à toxicidade de $\mathrm{Fe}$, mostrou o menor teor de $\mathrm{Fe}$ na matéria seca da parte aérea, confirmando resultados anteriores (CAMARGO, 1984).

Os resultados do presente trabalho demonstraram que os cultivares poderiam ser diferenciados em relação à tolerância à toxicidade de ferro e alumínio, empregando-se soluções com ou sem arejamento, desde que quantidades adequadas de Fe e Al sejam utilizadas nas soluções.

\section{SUMMARY}

\section{A COMPARATIVE STUDY OF THE TOLERANCE TO IRON AND ALUMINUM TOXICITY IN RICE}

Eleven rice cultivars were studied as the tolerance to iron and aluminum toxicity using seven different levels of these elements in the nutrient solutions with and without aeration under constant temperature $\left(30^{\circ} \mathrm{C} \pm 1^{\circ} \mathrm{C}\right)$ and $\mathrm{pH}=4.0$. The plant tolerance was measured taking into account the primary root length and the leaf and root dry weight of 20 plants of each cultivar after 10-day-growth in the different treatment solutions. With levels of 0,20 and $40 \mathrm{mg} / \mathrm{l}$ of $\mathrm{Al}$ in solutions with and without aeration and with the presence of $0.56 \mathrm{mg} / \mathrm{l}$ of $\mathrm{Fe}$ it was verified that the cultivars IAC-164, IAC-165, IAC-47, IAC-25, IAC-435, IAC-120, Blue Bonnet and Pérola were tolerant and the cultivars IR-8, IAC-899 and IR-841 were sensitive. When it was utilized $200 \mathrm{mg} / 1$ of $\mathrm{Fe}$ in aerated nutrient solution the cultivar IAC-164 showed high level of tolerance being significantly different from the cultivars IAC435, IAC-899, Blue Bonnet, IAC-25, IR-8 and IR-841. 'IAC-164' did not differ from the cultivars IAC-120, IAC-47, IAC-165 and Pérola. In non-aerated solution with the same concentrations of iron and aluminum, the same result was obtained with the difference that 'IAC-164' did not differ from 'IAC-25'. In aerated nutrient solutions containing $400 \mathrm{mg} / \mathrm{l}$ of $\mathrm{Fe}$ the most tolerant cultivar was IAC-164 which differed significantly only from IAC-899, IR-841 and IR-8. When non-aerated solution was used, no differences were found among the studied cultivars. 'IAC-164' presented high tolerance to $\mathrm{Al}$ and $\mathrm{Fe}$ toxicities even when it was applied $20 \mathrm{mg} / \mathrm{l}$ of Al plus $200 \mathrm{mg} / 1$ of $\mathrm{Fe}$ and $40 \mathrm{mg} / 1$ of $\mathrm{Al}$ plus $400 \mathrm{mg} / \mathrm{l}$ of $\mathrm{Fe}$ in the solutions. The Al and $\mathrm{K}$ concentrations increased and the $\mathrm{P}, \mathrm{Ca}, \mathrm{Mg}, \mathrm{Fe}, \mathrm{Cu}, \mathrm{Zn}$ and $\mathrm{Mn}$ concentrations decreased in the leaf dry matter for all aluminum tolerant cultivars, as the aluminum concentration in solution increased from 0 to $40 \mathrm{mg} / \mathrm{l}$. When the cultivars sensitive to Al toxicity were considered increasing levels of $\mathrm{Al}$ in solution caused an increase in $\mathrm{Al}, \mathrm{Fe}$ and $\mathrm{P}$ concentrations, and a decrease in the other element concentrations in the leaf dry matter. The $\mathrm{Fe}$ and $\mathrm{K}$ concentrations increased, $\mathrm{Ca}, \mathrm{Mg}, \mathrm{Mn}$, $\mathrm{Cu}$ and $\mathrm{Zn}$ concentrations decreased and no effect in $\mathrm{P}$ concentration was observed in the total leaf dry matter for all cultivars, when Fe concentration in nutrient solution increased from 0 to $400 \mathrm{mg} / 1$ in absence of $\mathrm{Al}$. The results showed that the studied rice cultivars could be separated in relation to $\mathrm{Al}$ and $\mathrm{Fe}$ toxicities in nutrient solution with and without aeration, since adequate quantities of $\mathrm{Al}$ and $\mathrm{Fe}$ are provided.

Index terms: iron toxicity in rice; aluminum toxicity in rice; aerated solutions; non aerated solutions; iron tolerance; iron sensitivity; aluminum tolerance; aluminum sensitivity; aluminum; iron; rice cultivars; tolerance; toxicity. 


\section{REFERENCIAS BIBLIOGRAFICAS}

ANNUAL REPORT FOR 1971. Los Baños, Philippines, International Rice Research Institute, 1972. p.40-41.

ANNUAL REPORT FOR 1972. Los Baños, Philippines, International Rice Research Institute, 1973. p.197.

ANNUAL REPORT FOR 1973. Los Baños, Philippines, International Rice Research Institute, 1974. p. 102.

ANNUAL REPORT FOR 1979. Los Baños, Philippines, International Rice Research Institute, 1980. p.118-119.

BATAGLIA, O.C.; TEIXEIRA, J.P.F.; FURLANI, P.R.; FURLANI, A.M.C. \& GALLO, J.R. Métodos de análise química de plantas. Campinas, Instituto Agronômico, 1978. 31p. (Circular, 87)

CAMARGO, C.E.O. Tolerância de cultivares de arroz em estádio de plântula a diferentes níveis de ferro em solução nutritiva. Bragantia, 43(2): 369$380,1984$.

; CAMARGO, O.B.A. \& SOUZA, D.M. diferentes concentrações de alumínio em solução nutritiva na tolerância de cultivares de arroz. Bragantia, Campinas, 43(2): 357-368, 1984.

MARTINES, C. Aluminum toxicity studies in rice (Oryza sativa L.). Corvallis, Universidade Estadual de Oregon, 1979. 113 fls. Tese. (Doutoramento) 